\title{
Tandem Self-Assembly of Block Copolymer: From Vesicles to Stacked Bowls
}

\author{
Xiaoqing Wang, ${ }^{\S a, b}$ Songlin Liu, ${ }^{\S c}$ Shida Cao, ${ }^{a}$ Fei Han, ${ }^{c}$ Hong Wang, ${ }^{* a}$ and Hongyu \\ Chen *a \\ ${ }^{a}$ Institute of Advanced Synthesis and School of Chemistry and Molecular Engineering, Jiangsu \\ National Synergetic Innovation Center for Advanced Materials, Nanjing Tech University, Nanjing \\ 211816, P. R. China Email: iashychen@njtech.edu.cn; ias_hwang@njtech.edu.cn \\ ${ }^{b}$ College of Science, Nanjing Forestry University, Nanjing 210037, P. R.China. \\ ${ }^{c}$ Division of Chemistry and Biological Chemistry, School of Physical and Mathematical Sciences, \\ Nanyang Technological University, 21 Nanyang Link, Singapore 637371 \\ ${ }^{\S}$ X. Wang and S. Liu contributed equally.
}

\section{Experimental section}

Acidity of deionized water. What needs to be explained is that the deionized water in our experiments measured with a $\mathrm{pH}$ meter to be 5.6 , which is close to $\mathrm{p} K_{\mathrm{a}}(5.25)$ of pyridine.

The yield of the aggregates. The aggregation yield is calculated as follows: Firstly, when the sample was characterized by TEM, the operator counted the numbers of all the products of various morphologies in the copper specimen grid. As shown in Figure 2, the sample included: vesicles ( 108), one chain (22 vesicles), two squashed vesicle chains (43 vesicles), four partially embedded bowl chains (98 bowls), twelve deeply stacked bowl chains (267 bowls), and one multi-layer structure (22 bowls). Then, defined as the number of aggregated vesicles/bowls over all vesicles/bowls, the yield of deeply stacked bowls $=\frac{267}{108+22+43+98+267+22} \times 100 \%=47.7 \%$. To obtain a more accurate result, the operator also counted those in the other two grids of the same sample. The yields were $42.8 \%$ and $43.6 \%$, respectively. Finally, the average yield of deeply stacked bowls was about $45 \%$. 


\section{Additional TEM/SEM images}

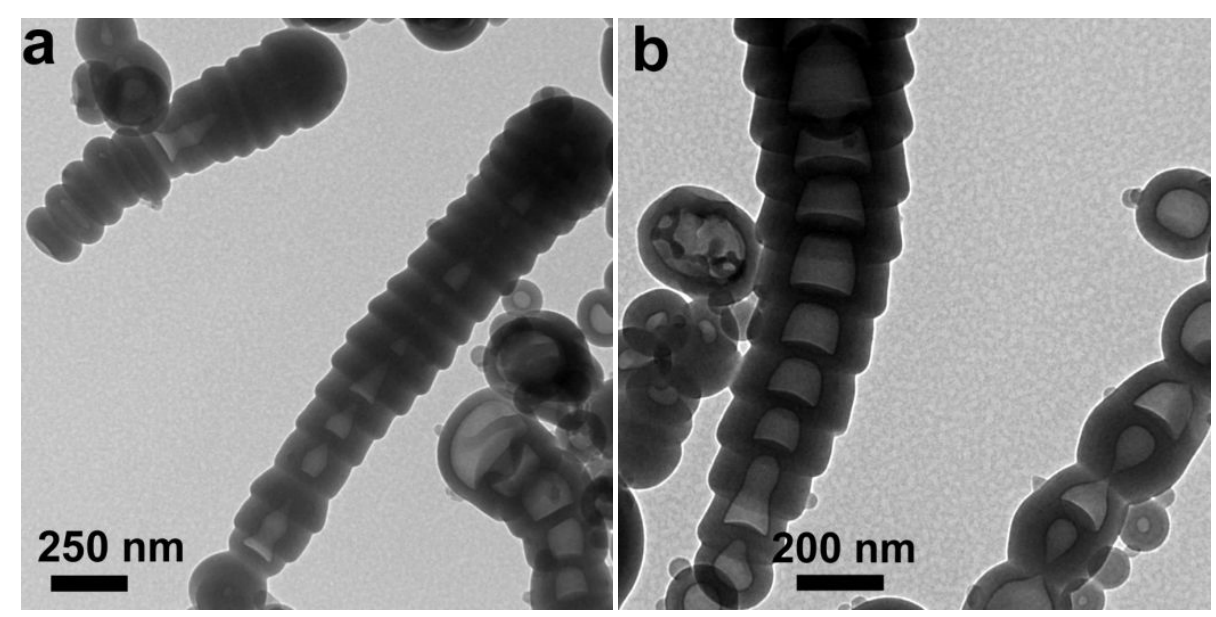

Figure S1. The morphologies of the stacked bowls. (a-b) TEM images of 1D chains of $\mathrm{PS}_{222}-b-\mathrm{P}_{4} \mathrm{VP}_{43}$ stacked bowls.

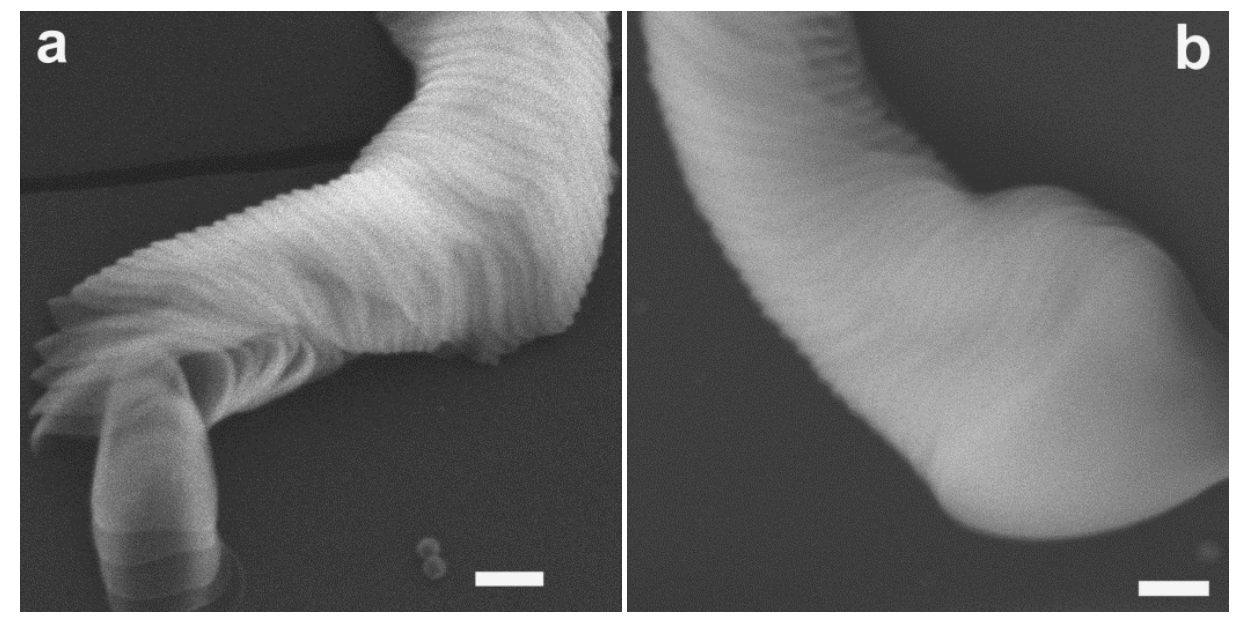

Figure S2. The morphologies of the multi-layers structures. (a-b) SEM images of the multi-layers structures incubated at $80{ }^{\circ} \mathrm{C}$ for $8 \mathrm{~h}$. Scale bar: $500 \mathrm{~nm}$ 

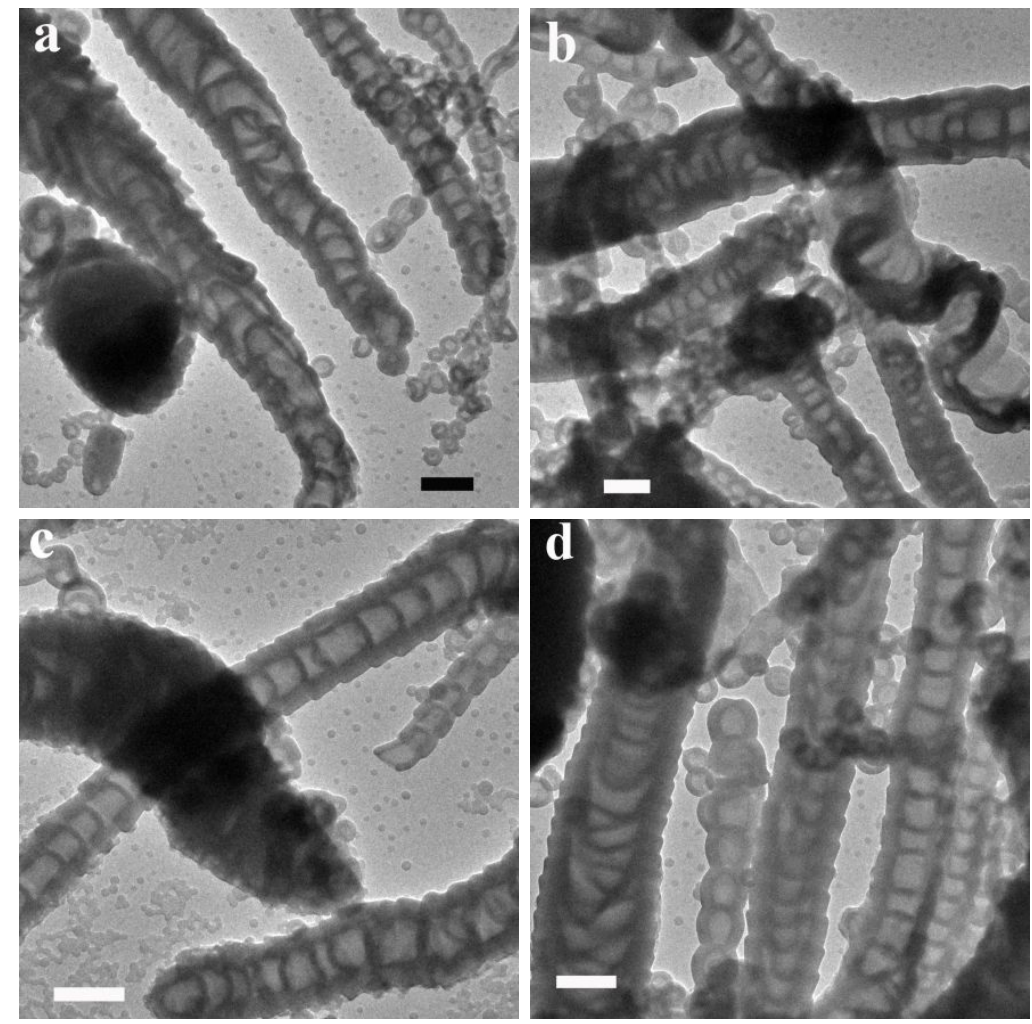

Figure S3. Multiple morphologies in a sample. (a-d) TEM images of the self-assembly structures incubated at $80{ }^{\circ} \mathrm{C}$ for $4 \mathrm{~h}$. Scale bar: $500 \mathrm{~nm}$

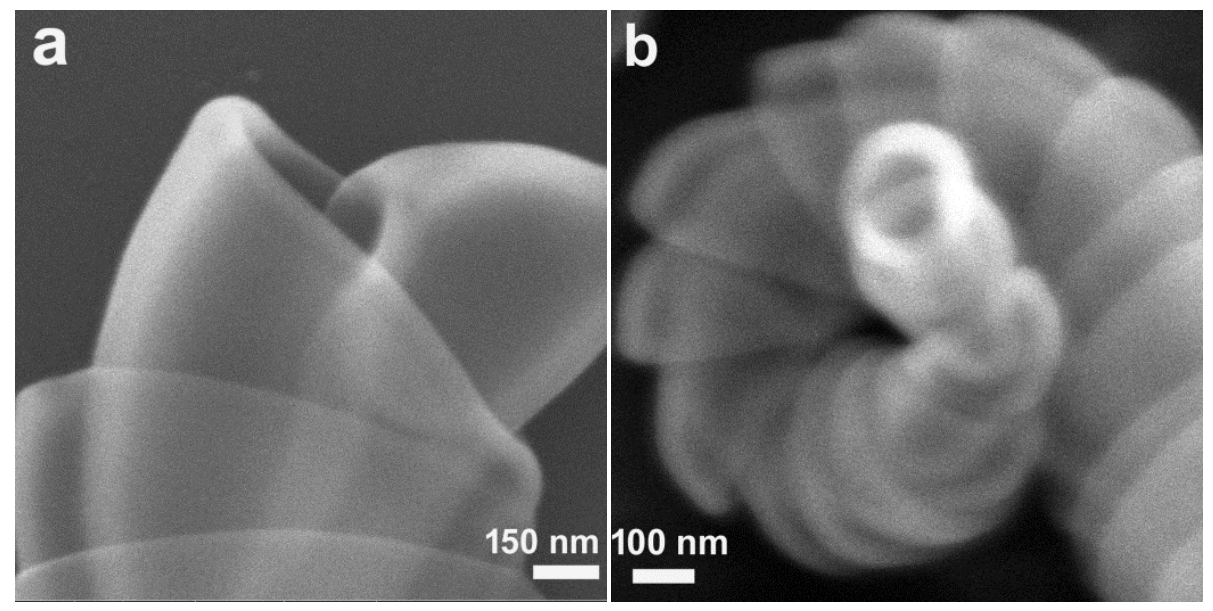

Figure S4. The openings of the bowls. (a-b) SEM images of the openings of bowls. 

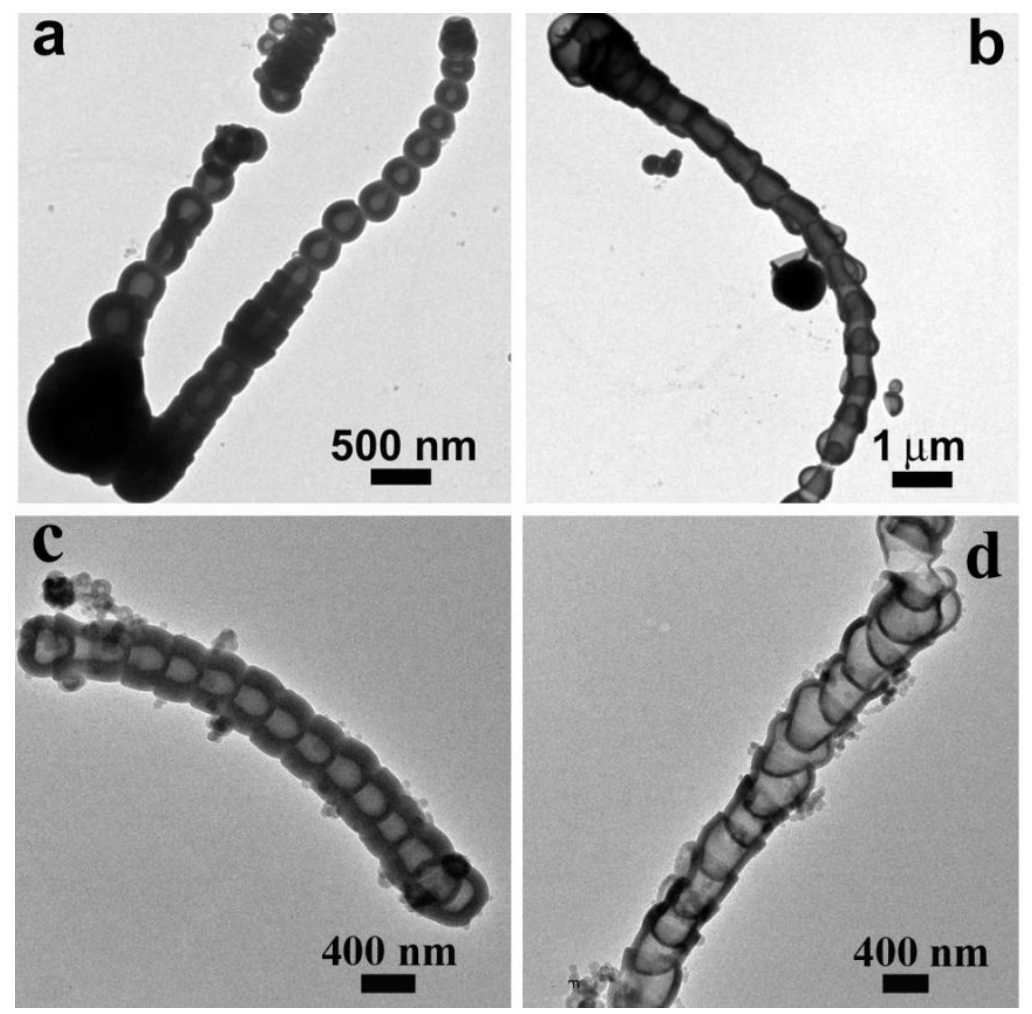

Figure S5. The structures upon basification. TEM images showing the products incubated at $80^{\circ} \mathrm{C}$ for $4 \mathrm{~h}$ and quenched by sodium hydroxide solutions (a, b) $\mathrm{pH}=6.7$; (c, d) $\mathrm{pH}=12.8$. 